\title{
Are absolute myocardial blood flow PET measurements ready for clinical use?
}

\author{
Ernest V. Garcia
}

doi: $10.1007 / s 12350-014-9969-2$

As a preamble let us remind ourselves that nuclear cardiology continues to be deeply rooted in the study of cardiovascular physiology. Our images are inherently digital and quantitative where a pixel value, through the tracer principle, represents in somewhat direct proportion a cardiovascular physiological parameter such as myocardial perfusion.

By the enthusiasm demonstrated at ASNC's recent PET Summit in Baltimore, our field seems to be finally poised to welcome cardiac PET as an important tool to complement our imaging armamentarium and perhaps more importantly to propel our assessment of physiology to a new level. Those of us that have seen PET's continuous progress for the last 30 years realize that acceptance by our field has been no easy task since our peers seem to mistrust and fear what they do not know, and many did not have and thus not know cardiac PET first hand. Kudos are definitely owed to our cardiac PET pioneers such as Lance Gould and Heinz Schelbert, not only for their contributions as physician-scientists but perhaps even more laudable for their drive to persevere in their quest to bring us cardiac PET. This quest has been taken up by the likes of Marcelo Di Carli's, Rob Beanlands', and Tim Bateman's laboratories. Many important contributors are missing from this list, both within and outside the United States, but what the names above have in common beyond their scientific contributions is their level of effort in developing, validating, implementing the tools, and establishing the clinical applications associated with the PET measurement of absolute myocardial blood flow and myocardial flow reserve. This brings us to the title of this editor's page: Are absolute myocardial blood flow measurements ready for clinical use? The answer lies in the answer to the other questions below.

J Nucl Cardiol 2014;21:857-8.

1071-3581/\$34.00

Copyright (C) 2014 American Society of Nuclear Cardiology.
Perhaps in a patient-centered environment the first pertinent question should be how will these measurements help our patients? To paraphrase what Lance Gould so eloquently explained at the PET Summit, we should not limit the role of the PET measurements of myocardial flow and flow reserve as a gatekeeper to the catheterization laboratory but more importantly as a gatekeeper to revascularization. So not only should this approach limit the referral of the $60 \%$ of patients who are found not to have obstructive disease in the catheterization laboratory but for the $40 \%$ with obstructive disease guide the interventionist as to which vessels are truly flow limiting and have the potential for successful revascularization. As such, the measurements fulfill the mantra of today, i.e., an imaging test should not only have the attribute of yielding a correct diagnosis but should also guide a successful therapy thus being directly associated with a patient's outcome that can be used as evidence of the value of the test.

The next pertinent question should be: with today's instrumentation, radiopharmaceuticals, and quantification software-are the measurements of absolute flow and flow reserve accurate and reproducible enough for clinical use? The simplistic but pertinent answer is that for a given clinical application, in a given patient, if the error of the measurement is such that we can reasonably separate, in our case, normal flow and/or flow reserve from abnormal flow and/or abnormal flow reserve then the technique is ready for clinical use. The more convoluted answer is that we should remind ourselves of the differences between efficacy and effectiveness as well as try to avoid the mistakes of the past when introducing new technology. The efficacy of these flow measurements are well established. This is true particularly for flow reserve with $\mathrm{N}-13$-ammonia, $\mathrm{Rb}-82$ and $\mathrm{O} 15$-water. Thus, the accuracy and reproducibility of the "methods" have been shown to work well in clinical trials and laboratory studies, usually in the hand of experts. The effectiveness of these flow measurements are not yet established. Thus what is not established to date is how well these measurements work in the daily practice of 
medicine to produce a positive outcome using measurements from a typical nuclear cardiology laboratory. Clearly there is always room for further improving the clinical value of these measurements such as better separating the flow impairment due to microvascular disease vs epicardial disease or being able to assign the flow impairment to a specific vessel branch.

This brings to light the mistakes of our past when introducing new technology. Two technologies come to mind, first-pass radionuclide angiography and SPECT attenuation correction. Even though most experts would agree that both of these techniques have been shown to be highly efficacious the frequency of their current use in patient studies is disappointing. The limiting factor in the use of first-pass studies was the complaint that it was too difficult to perform, in part because of the need for a bolus injection, synchronized start of the acquisition, fast counting cameras, fast framing rates, and so on. The initial complain about SPECT attenuation correction was that it did not work, i.e., reduced the diagnostic yield. Later when the methodology matured and shown to work in the daily routine the complaint was that there was no reimbursement for this additional task. These technologies shared the common mistake of rushing to clinical use technology before they were ready. The readiness factors include the commercial availability of the right equipment, right radiopharmaceutical, easy to use and robust software, and perhaps most important appropriate training for the users. As we learned with SPECT attenuation correction, once a new technology develops a bad reputation it takes years to create the trust for clinical use, even after all the technical problems have been fixed. Moreover, had these techniques obtained wide acceptance, MBF, and flow reserve could be measured with SPECT. These flow measurements would require first-pass techniques to capture the input function, SPECT attenuation correction for measuring absolute concentration, cardiac-centric high count sensitivity cameras to reduce noise, SPECT tracers with higher extraction fraction (such as teboroxime and I-123 rotenone), and flow software similar to that used in PET.

Today in PET we have a mature, sophisticated imaging technology, well capable of imaging for flow measurements. We also have adequate tracers and robust software capable and validated to generate accurate and reproducible flow measurements. Scientists like Rob deKemp and Piotr Slomka are collaborating to make sure that the various software packages generate reproducible methods for quantification of myocardial blood flow and flow reserve to facilitate the exportability of clinical results, multicenter and registry trials, and metaanalysis of outcomes. Importantly, their efforts these days is in demonstrating how similar the flow results can be rather than in the past where the emphasis was in showing how different ejection fraction and perfusion results were. Regarding reimbursement, the new mantra is value, if we manage the cost of the test, the quality of the results commensurate with patient outcomes will continue to drive the nuclear cardiology field. The one area where our field appears to be lacking as it relates to PET myocardial blood flow and flow reserve measurements seems to be in training both our fellows as well as practicing nuclear cardiologists. Hopefully this editorial will help motivate us to do more.

Finally, the answer to the posed question, Are absolute myocardial blood flow PET measurements ready for clinical use?, lies in the form of the advice given by President Ronald Reagan: "Trust, but verify.", Trust that the measurements of MBF and flow reserve by PET are reliable but perform additional research in your clinic to verify that in your laboratory the results are accurate, clinically useful, and trustworthy. 